\title{
Novel Uses of EMG to Study Normal and Disordered Motor Control
}

\author{
Richard B. Stein
}

\begin{abstract}
The electromyogram(EMG) is unique in that it gives the output of a single class of neurons, $a$-motoneurons, in a way that can be readily recorded and studied in normal subjects as well as in patients with a variety of disorders. Although the EMG has been used for many years, new applications continue to be found. This paper reviews in succession four recent approaches to EMG recording that have provided insight into the function of the normal and the disordered nervous system.
\end{abstract}

RÉSUMÉ: Utilisations inédites de l'EMG pour l'étude du contrôle moteur normal et pathologique L'électromyogramme (EMG) est unique en ce qu'il donne le signal d'une seule classe de neurones, les $a$-motoneurones, d'une façon qui peut être facilement enregistrée et étudiée chez des sujets normaux ainsi que chez des patients atteints de pathologies variées. Même si l'EMG est utilisé depuis plusieurs années, des applications nouvelles continuent d'être découvertes. Dans cet article nous revovons successivement quatre approches récentes, utilisant l'enregistrement électromyographique, qui ont contribué à une meilleure connaissance du fonctionnement du système nerveux normal et pathologique.

Can. J. Neurol. Sci. 1988; 15:95-98

\section{EMG in Response to SynChronous Stimulation During Behaviour}

Synchronous stimulation of motor nerves is routinely used, for example, in the measurement of nerve conduction velocity. Stimulation of some nerves, such as the tibial nerve behind the knee (popliteal fossa) is well known to produce two responses, as shown in the top trace of Figure 1. A motor or M-wave results from direct stimulation of $a$-motoneurons and a Hoffman or H-reflex results from stimulation of primary muscle spindle afferents (Group Ia fibres) which have monosynaptic connections to the $a$ motoneurons.

This dual response has permitted us to study the modulation of reflex responses in a variety of normal behavioural tasks such as standing, walking and running. ${ }^{1,2} \mathrm{~A}$ problem, which has precluded previous studies of $\mathrm{H}$-reflexes during movement, is that the surface electrodes can move with respect to the underlying nerve. Thus, change in the EMG response could be due to an artefact of electrode movement, as well as to any central changes in the reflex pathway.

We overcame this problem simply by repeating the experiment at a variety of stimulus strengths and choosing values among the various stimuli used that produced nearly the same M-wave on average at each phase of the step cycle. We reasoned that if a roughly constant percentage of $a$-motoneurons were being stimulated, then a roughly constant percentage of Ia afferents were probably being stimulated as well. Changes in the $\mathrm{H}$-reflex at a constant $\mathrm{M}$-wave would then represent changes in the central part of the reflex pathway (i.e., in synaptic transmission).

Figure 1 shows results of an experiment in which a normal subject was walking on a treadmill. The step cycle was divided into sixteen parts, starting at the time the heel contacted the ground. Stimuli which occurred in each part were averaged separately. The $\mathrm{H}$-wave recorded over the soleus muscle increased progressively during the stance phase (when the foot was on the ground) and was small during the swing phase at the end of the cycle (when the foot was moving through the air). Clearly, the reflex is being modulated substantially as part of the normal patterning of human locomotion.

\section{Correlations With Asynchronous EMG ACtivity}

To understand the mechanism of this modulation it is necessary to compare the reflex modulation with changes in the number of active motoneurons. The larger reflex might simply arise from the fact that more motoneurons are actively participating in the movement or are close to their threshold for firing. A common measure of motor activity is obtained by rectifying and integrating the surface EMG. 


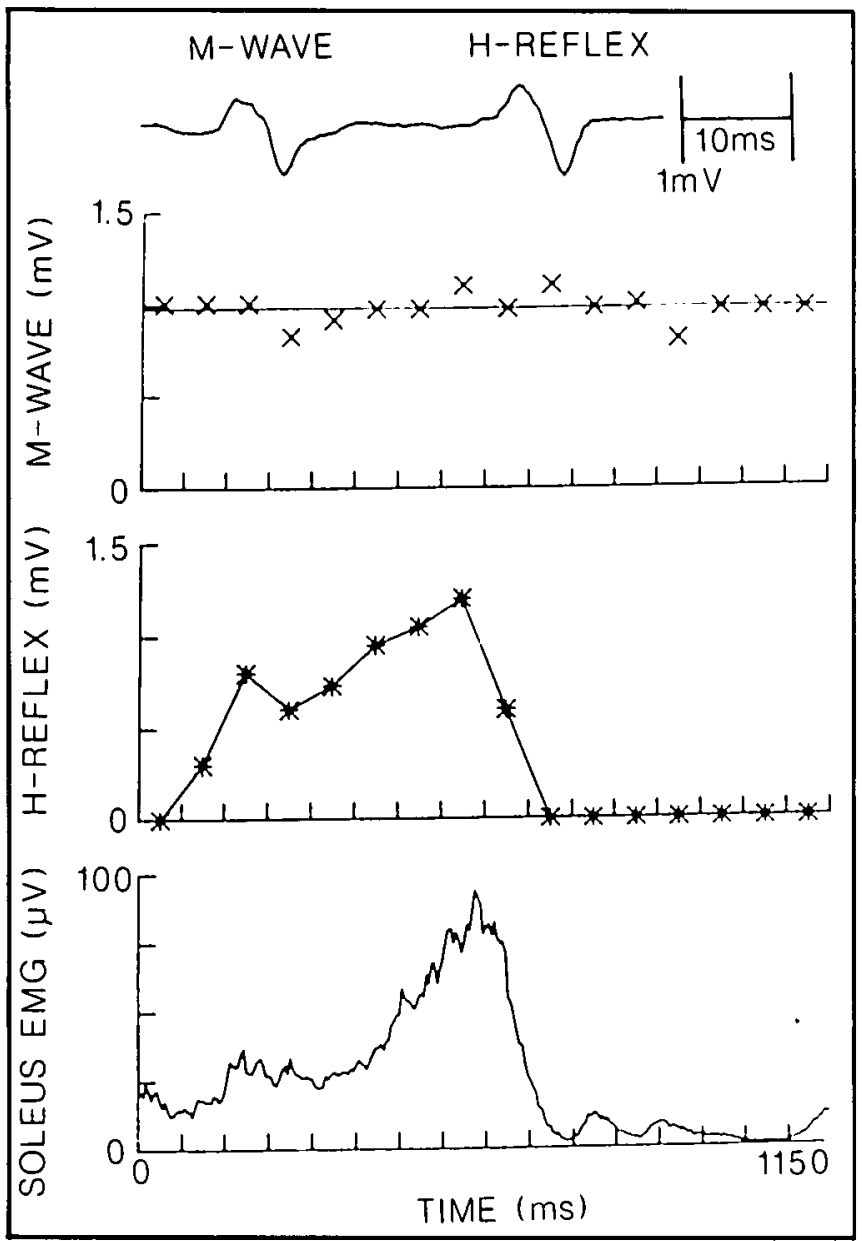

Figure $I-M$-waves and $H$-reflexes were recorded to tibial nerve stimulation at various phases of the step cycle starting at heel contact. At a relatively constant level of $M$-wave the $H$-reflex increased as the soleus EMG increased during the stance phase of the step cycle and decreased during the swing phase.

Figure 1 also compares the modulated $\mathrm{H}$-reflex with the rectified and integrated surface EMG from the soleus muscle in cycles where no stimuli were applied. The H-reflex increases approximately in parallel with the activity in the soleus motor pool. However, neither the $\mathrm{H}$-wave nor the integrated surface EMG is a linear measure of the number of active motoneurons. Larger motoneurons innervate more and larger muscle fibres so the $\mathrm{H}$-wave will reflect these differences in innervation. In addition, the rectified and integrated surface EMG will be influenced by the firing rates of each motoneuron and the superposition of the unit potentials will produce some cancellation. Nonetheless, the integrated surface EMG often varies approximately linearly with the level of force produced by the muscle under isometric conditions ${ }^{3}$ for reasons that probably involve the cancellation of several types of nonlinearity. ${ }^{4}$

Figure 2 compares the modulation of the H-reflex during walking with that during standing. Subjects were asked to adjust the amount of tonic activity in the soleus muscle to generate various levels of surface EMG. A voltmeter was used to display the rectified and integrated surface EMG, a simple form of biofeedback. Although the H-reflex increased approximately in parallel with the level of activity during walking, it was already large during quiet standing and increased little with increased levels of tonic activity. The functional implications of the differential control of the reflex during the two types of behaviour have been discussed elsewhere, ' as well as possible synaptic mechanisms. ${ }^{5}$ We simply wish to point out here that these results indicate that even this simple, largely monosynaptic reflex can be modified adaptively during the performance of various common tasks.

More complex reflexes may also be studied using the asynchronous EMG activity, as shown in Figure 3. In this example, the common peroneal nerve was stimulated to produce disynaptic inhibition of the ongoing soleus activity (see inset). The amount of inhibition could also be studied during various motor tasks. In contrast to the monosynaptic reflex, the disynaptic inhibition increased approximately linearly with the EMG level, during both walking and standing. Use of the surface EMG to study these reflexes nicely complements results obtained from single motor unit studies with needle electrodes. ${ }^{6}$

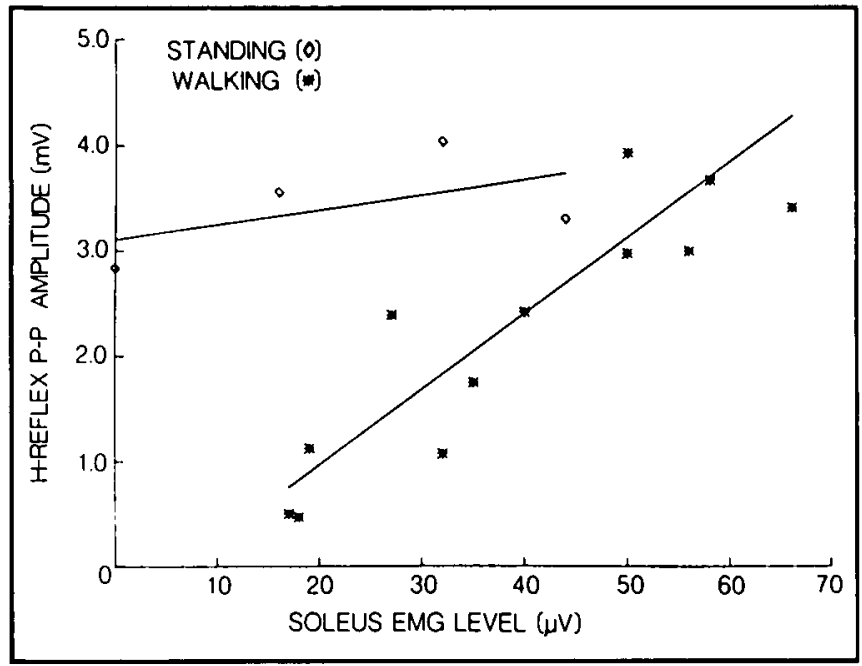

Figure 2 - The H-reflexis modulated differentlyduring walking and standing, particularly at low levels of muscle activity as measured by the surface EMG. From Capaday \& Stein.'

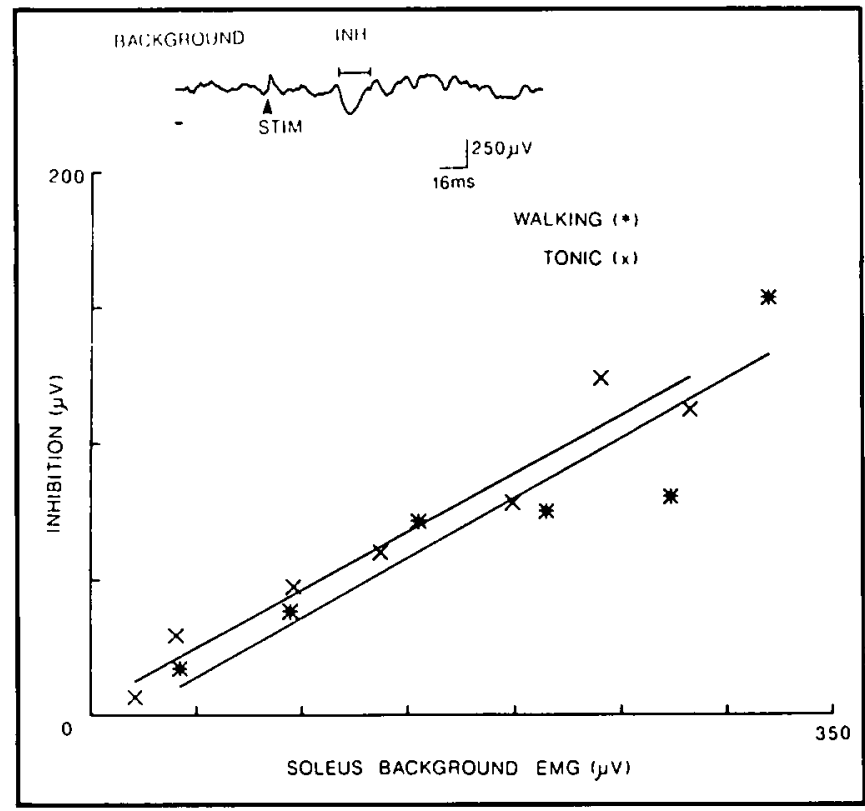

Figure 3 - Stimulation of the common peroneal nerve produced a briefinhibition of the background level of excitation measured by the surface EMG. The level of inhibition varied linearly with the level of excitation both in walking and standing. 


\section{Spike-Triggered Averaging of NeEdle EMG to Study MOTOR UNIT FORCE}

I would like to turn now to the use of needles inserted into a muscle (needle EMG) to study the properties of single motor units in more detail. Correlation of the activity with records of the surface EMG or muscle force can often provide important extra information not available from the needle recording alone.

Figure 4 shows needle and surface records taken from a patient whose ulnar nerve had been severed at the wrist and the nerve regenerated back to the several hand muscles innervated by that nerve. ${ }^{7}$ The needle was inserted into the abductor digiti minimi (ADM) muscle and a motor unit fired briskly when the subject activated this muscle but not other ulnar innervated muscles, such as the first dorsal interosseus (FDI) or adductor pollicis (AP) muscles. This unit had apparently grown back to the muscle it innervated prior to the accident.

However, when the subject activated the other muscles, substantial surface EMG activity was seen, despite the fact that these muscles were on the other side of the hand and direct spread of electrical activity could be ruled out. The cause of this activity was that many motor units had been misdirected during regeneration; i.e., they had grown back to a muscle different from the one they had originally innervated, as shown in Figure 4b. Another unit recorded with the needle fired when the subject moved his first finger to activate the FDI muscle, but not when he moved the thumb to activate the AP muscle or the little finger so as to activate the ADM muscle where the needle had been inserted.

Misdirection of motor units was so common that motoneurons appeared to enter and regenerate through Schwann cell sheaths almost at random. When there were several choices, such as in the case of the ulnar innervated muscles, many motoneurons ended up in inappropriate muscles. This mis-

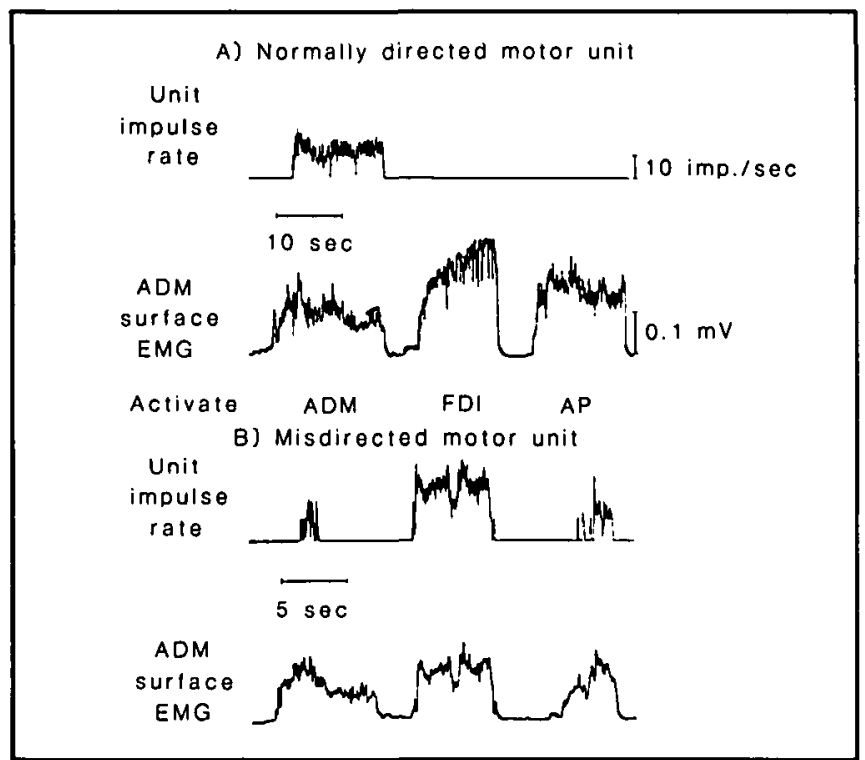

Figure 4 - Impulse rate of single motor units which were normally directed $(A)$ and misdirected $(B)$ following ulnar nerve section and reinnervation of the abductor digiti minimi ( $A D M$ ) muscle. Surface EMG was also recorded from the $A D M$ muscle when the subject was asked to activate either the $A D M$, the first dorsal interosseus (FDI) or the adductor pollicis (AP). Substantial activity was observed when the subject attempted to activate any of these muscles, indicating that many of the units were misdirected during reinnervation. From Thomas et al. ${ }^{\text {? }}$ direction of motoneurons probably explains the lack of dexterity that commonly results from ulnar nerve section even when the surgical repair was successful in other respects.

Figure 5 shows another consequence of misdirection of motor units, this time resulting from section of the median nerve. In a normal human muscle (Figure SA) there is an orderly recruitment of motor units during an increasing voluntary contraction in order of their size. ${ }^{8}$ This is an example of the size principle of Henneman, ${ }^{9}$ which was first studied in the reflex responses of motoneurons in experimental animals.

The contractile force of the human motor units was estimated from the force fluctuations correlated with the discharge of single motor units, ${ }^{10}$ a technique now known as spike-triggered averaging. In a patient who had severed her median nerve at the wrist, the size principle returned very well. In another patient, who had severed his nerve at the elbow, the recruitment of motor units appeared to be random.

This difference was consistently observed and can be most easily explained on a simple anatomical basis. The median nerve at the wrist contains motoneurons which supply only a few, largely synergistic muscles, so misdirection can not affect function appreciably. However, the median nerve at the elbow contains motoneurons which innervate many muscles. Measuring the threshold force of thumb abduction required to activate the motoneuron would not be an appropriate measure of size if it innervated a muscle with a quite different function.

\section{Use OF Microelectrodes to Measure Motor Unit ConduCtion Velocity}

Clearly, a more direct measure of motoneuron size is needed to study the size relationships in patients where some misdirection of motor units has occurred. Since conduction velocity is directly correlated with axon diameter, a technique for measur-

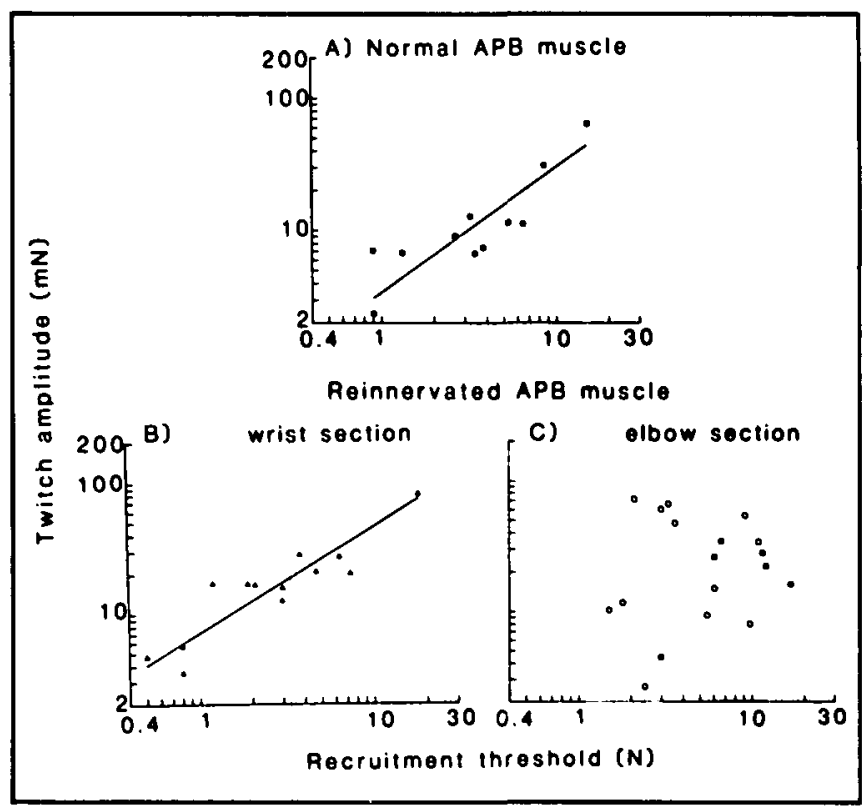

Figure $5-$ Motor unit twitch amplitudes versus recruitment thresholds for a normally innervated abductor pollicis brevis $(A P B)$ muscle $(A)$ and for muscles which were reinnervated after section of the median nerve at the wrist $(B)$ or the elbow $(C)$. Note the absence of any trend after the nerve was sectioned at the elbow. Modified from Thomas et al.? 


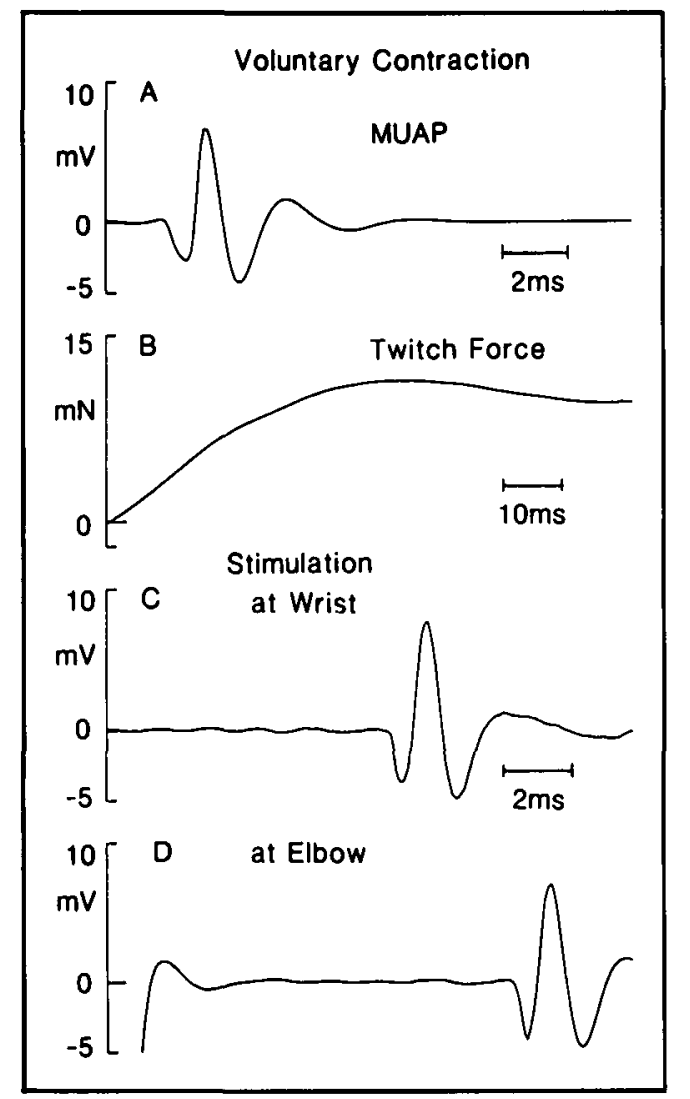

Figure 6 - Spike-triggered averaging was used to estimate the twitch force $(B)$ produced by a single motor unit action potential (MUAP) that was recorded with a microelectrode $(A)$. Under favourable conditions the same unit could be isolated following stimulation of the whole median nerve at the wrist $(C)$ and the elbow $(D)$ because of the extreme selectivity of the electrode. The conduction velocity of the single motor axon could be calculated from the difference in latency in C and D. From Dengler et al.

ing the conduction velocity of single motor units would be highly desirable.

Figure 6 shows results using a recently developed technique. ${ }^{11}$ Rather than using a conventional needle, a microelectrode of the type used for microneurography is inserted into the muscle. The microelectrode is so selective that it records from only a very few muscle fibres. The voluntary activity of one unit is used for spike-triggered averaging as shown in the Figure. Then, the nerve can be stimulated at the wrist and the elbow. In about a quarter of the units studied, the same unit could be selectively activated from both sites and therefore the conduction velocity of the single motor unit could be determined.

For another quarter of the units a second unit was the first one to be stimulated, but computer subtraction techniques allowed the extraction of the unit of interest. ${ }^{11}$ For the other half of the units, the single unit potential could not be extracted with confidence for stimulation at both sites. Nonetheless, the yield was sufficiently good that the technique should find application in a number of clinical situations.

In conclusion, although EMG has been used as a tool for many years, scope still exists for expanding its use in novel ways for basic studies on motor control and for clinical applications.

\section{ACKNOWLEDGEMENTS}

I appreciate the many essential contributions of students and colleagues to the original studies (see Reference list), which have been summarized here. These studies were supported in part by the Medical Research Council of Canada and the Muscular Dystrophy Association of Canada.

\section{REFERENCES}

1. Capaday C, Stein RB. Amplitude modulation of the soleus H-reflex in the human during walking and standing. J Neurosci 1986; 6 : 1308-1313.

2. Capaday C, Stein RB. Differential modulation of the human soleus H-reflex during walking and running. J Physiol 1987; 392:513-522.

3. Lippold $\mathrm{OCJ}$. The relation between integrated action potentials in a human muscle and its isometric tension. J Physiol 1952; 117: 492-499.

4. Milner-Brown HS, Stein RB. The relation between the surface electromyogram and muscular force. J Physiol 1975;246: 549-569.

5. Capaday C, Stein RB. A theoretical method for the analysis of the reflex output of a motoneuron pool. J Neurosci Methods 1987; 21: 91-104.

6. Ashby P, LaBelle K. Effects of extensor and flexor group I afferent volleys on the excitability of individual soleus motoneurons in man. J Neurol Neurosurg Psychiatry 1977; 40: 910-919.

7. Thomas CK, Stein RB, Gordon T, Lee RG, Elleker MG. Patterns of reinnervation and motor unit recruitment in human hand muscles after complete ulnar and median nerve section and resuture. J Neurol Neurosurg Psychiatry 1987; 50: 259-268.

8. Milner-Brown HS, Stein HB, Yemm R. The orderly recruitment of human motor units during voluntary isometric contractions. J Physiol 1973; 230: 359-370.

9. Henneman E, Mendell LM. Functional organization of motoneuron pool and its inputs. In: Brooks VB, ed. Handbook of Physiology; Sect. 1, The Nervous System; Vol. 2, Motor Control. American Physiological Society, Bethesda, MD 1981; 423-507.

10. Milner-Brown HS, Stein RB, Yemm R. The contractile properties of human motor units during voluntary isometric contractions. J Physiol 1973; 228: 285-306.

11. Dengler R, Stein RB, Thomas CK. Axonal conduction velocity and force of single human motor units. Muscle Nerve 1987 (in press). 